\title{
A STUDY OF STOOL SAMPLE FROM HIV POSITIVE AND HIV NEGATIVE AT ANDHRA PRADESH
}

\author{
GUNDALA OBULESU ${ }^{1 *}$, HANUMANTHAPPA AR ${ }^{2}$, PRABAKAR REDDY E ${ }^{3}$
}

${ }^{1} \mathrm{PhD}$ scholar, Bharath University, Chennai, Tamil Nadu, India. ${ }^{2} \mathrm{PhD}$ scholar, J.J.M. Medical College, Davangere, Karnataka, India. ${ }^{3}$ Department of Biochemistry, Sree Lakshmi Narayana Institute of Medical Sciences, Bharath University, Pondicherry, India. Email: obulesu100@gmail.com

Received: 25 April 2017, Revised and Accepted: 21 November 2017

ABSTRACT

Objective: The objective of this study is to investigate the variations, if any, in the types of intestinal parasites in HIV-positive and HIV-negative individuals.

Methods: Study group 75 HIV positive cases, Control group HIV non reactive 51, total 125 faecal sample examined For HIV conformation screening test like TRI DOT, ELISA, for conformation HIV by using PCR, for stool examination used by following test like microscopic examination, formal ether concentration technique, saline wet mount,iodine wet mount, formal ethe sedimentation , modified acid fast stain.

Results: For the purpose of statistical analysis, the enteric parasites are divided into the coccidian group (Cryptosporidium+Isospora belli) and conventional enteric parasites (Entamoeba histolytica, Giardia intestinalis, and Ascaris lumbricoides), and enteric parasitic infection is more common in HIV-positive patients with diarrhea than HIV-positive patient without diarrhea or HIV-negative individuals.

Conclusion: The study period of 1 year and 8 months revealed that it is difficult to locate/identify a HIV-positive person in a place like Kadapa on account of confidentiality/lack of awareness among the population. Detection of intestinal parasites is $17.3 \%$ in HIV-positive patients and $12 \%$ in HIV-negative individuals.

Keywords: Acquired immunodeficiency syndrome, HIV, Parasite.

(C) 2018 The Authors. Published by Innovare Academic Sciences Pvt Ltd. This is an open access article under the CC BY license (http://creativecommons. org/licenses/by/4. 0/) DOI: http://dx.doi.org/10.22159/ajpcr.2018.v11i1.19377

\section{INTRODUCTION}

Since the original description of acquired immunodeficiency syndrome (AIDS) in 1981 by Michael Gottlieb et al. in Losangeles, USA, a wide variety of gastrointestinal manifestations mainly opportunistic enteric parasitic infestations are described in patients with HIV infection [1]. Diarrhea is a common clinical manifestation of these infections. Identification of the etiological agent of diarrhea in HIV patient is very important as it can help in the institution of appropriate therapy and reduction of morbidity and mortality [2]

It has been estimated that $30-50 \%$ of patients with AIDS in the USA and about $90 \%$ in Africa and Haiti suffer from chronic diarrhea [3]. Infection caused by different pathogens cannot be differentiated clinically unless specific fecal examination is carried out. The isolation rates of intestinal parasites in patients with AIDS and chronic diarrhea vary from $40 \%$ to $83 \%$, and the parasitic agents also differ markedly from region to region [3]. Until AIDS epidemic, several of the presently recognized parasitic infections were almost unknown as the cause of human diseases [1].

Diarrhea is the presenting symptom of approximately a third of patients with HIV infection. Chronic diarrhea significantly reduces the quality of life in patients with HIV infection and is an independent predictor of mortality in AIDS. A variety of enteric pathogens have been isolated from AIDS patients with diarrhea, but it is not clear that these enteric infections are necessarily associated with the presence of diarrhea [4]. The diarrhea wasting syndrome in association with a positive HIV serology test is an AIDS-defining illness in the World Health Organization (WHO)'s classification [5]. Cryptosporidium and Isospora belli, two of the intestinal coccidian parasites known to be the causative agents of diarrhea in animals, have now emerged as one of the main causes of prolonged life-threatening diarrhea in immunocompromised hosts particularly so in patients with AIDS [6]. Intestinal microsporidiosis and cyclosporiasis have also been reported with increasing frequency in HIV-infected patients [7]. In HIV-infected patients, progressive decline in their immunological responses makes them extremely susceptible to a variety of common and opportunistic infections.

\section{METHODS}

The present study was conducted from July 2014 to December 2016 FIMS, Kadapa.

\section{Study group}

Seventy-five HIV-reactive (positive) patients (age 15-59 years) were identified with the help of clinicians of RIMS and FIMS. These patients were being treated by the clinicians for recurring ailments. The study group comprised of 55 male and 20 female HIV-positive patients.

\section{Control group}

Fifty HIV non-reactive individuals (34 male and 16 female), aged 18-60 years, admitted in RIMS and FIMS, Kadapa, for various ailments in surgical wards were included in the study as a control group. Stool samples were collected from these patients after collecting relevant information such as age, sex, occupation, present complaint, whether on antiretroviral therapy (ART), and treatment given. Both diarrheic and non-diarrheic stool samples were collected for parasitological examination.

Total number of fecal samples examined $=125$

\section{Laboratory diagnosis of HIV infection}

The HIV-positive cases included in this study were identified through the clinicians, who were treating them for their recurrent ailments. They were investigated and identified as HIV reactive by serological tests done elsewhere. All of the patients were reported enzyme-linked immunoasorbant assay (ELISA) positive (as per WHO strategy-II 1993) for HIV-1/HIV-2 with at least two different kits [1]. 
The individuals included under the control group were screened for their HIV status in our laboratory by ELISA and TRIDOT [7]. Method during admission into surgical wards at RIMS and FIMs, during July 2014 to December 2016.

\section{Collection of samples}

Fecal sample

From each patient, one freshly voided stool sample was collected in a clean, wide-mouth sterile container and examined within $2-3 \mathrm{~h}$ of collection.

\section{Processing of samples}

Fecal sample

Naked eye examination: Color, consistency, and presence of any parasitic forms in the stool were noted.

\section{Microscopic examination}

Each sample was subjected to microscopic examination for the presence of any protozoal cyst or trophozoites, helminthic ova or larvae, or any other relevant findings. Saline mount and iodine (Lugol's) wet mount were prepared and examined under the microscope using $10 \times$ and $40 \times$ objectives and findings were noted.

\section{Formal-ether concentration technique for parasites}

Each stool sample was subjected to formal-ether concentration technique, and the concentrated sediment is examined by saline wet mount and iodine wet mount to detect the parasitic forms. Thin smear is prepared from the deposit, heat fixed, and stained by modified Ziehl-Neelsen staining technique. Procedures were according to the standard methods mentioned in the appendix. [Saline wet mount, iodine wet mount, formal ether concentration technique, and modified Z-N staining]. Microscopic pictures of the parasitic forms were taken on the spot, with the binocular microscope MOTIC B-1 series with inbuilt digital camera, available in the Department of Microbiology, Fathima Institute of Medical Sciences, Kadapa, Andhra Pradesh.

\section{Saline wet mount}

Saline wet mount is made by mixing a small quantity of feces with a drop of physiological saline. It is seen under low- and high-power microscopy. It is used to demonstrate helminthic eggs and larvae. It is also used to detect motile trophozoites of the intestinal protozoa [8].

\section{Iodine wet mount}

Iodine wet mount is made using a drop of iodine for preparation of wet mount of stool. Dobell and O'Connor's and Lugols' and D'Antonie's iodine are different types of iodine frequently used in the iodine wet mount. Iodine wet mount is mainly used for protozoal cysts. Iodine stained cysts show pale refractile nuclei, yellowish cytoplasm, and brown glycogen material. The chromatoidal bodies are not clearly visible. The motility of trophozoites is inhibited in iodine wet mount $[9,10]$.

\section{Formalin ether sedimentation method for parasites [9]}

The advantage of this method is that formalin fixes the eggs, larvae, and cysts so that they become non-infectious. The procedure consists of the following steps:

- Half teaspoon of fresh stool is taken in a $15 \mathrm{ml} \mathrm{screw-capped} \mathrm{test} \mathrm{tube.}$ It is mixed with $10 \%$ formalin and is allowed to stand for $30 \mathrm{~min}$.

- The fecal suspension is filtered through two layers of gauze in a funnel into the centrifuge tube. Saline is added to the tube to bring the fluid level within several millimeters of the rim of the tube. The tube is centrifuged for $10 \mathrm{~min}$ at $500 \mathrm{~g}$

- The supernatant is discarded. The sediment is resuspended in saline and again centrifuged for $10 \mathrm{~min}$ at $500 \mathrm{~g}$.

- The sediment is resuspended in $7 \mathrm{ml}$ of $10 \%$ formalin. $3 \mathrm{ml}$ of ether or ether substitute is added to it. The tube is closed with a stopper and shaken vigorously for $30 \mathrm{~s}$. The tube is held in such a way that the stopper is held away from the face. The stopper is removed carefully.

- The tube is centrifuged for $10 \mathrm{~min}$ at $500 \mathrm{~g}$. The tube is then allowed to stand in a test tube stand. Four layers are formed. The first bottom layer is the sediment which contains parasitic forms, second layer is the layer of formalin, third is the layer of fecal debris, and top is the layer of ether or ether substitute.

- The layer of fecal debris is removed by an applicator stick from the site of the tube, and then, all the liquid is decanted leaving behind a drop of formalin and the sediment. The sediment is mixed with formalin drop, and either a wet mount is prepared for microscopic examination or a smear is made for modified Z-N staining.

\section{Modified acid-fast stain $[9,10]$}

The modified acid-fast stain is now increasingly being used for the detection and identification of Cryptosporidium, Isospora, and Cyclospora oocysts. Both hot and cold modified acid-fast stains have been used with equal sensitivity. The staining solution contains:

1. Carbol fuschin: It contains basic fuschin $4 \mathrm{~g}$; phenol $8 \mathrm{ml}$; alcohol (95\%) $20 \mathrm{ml}$; and distilled water $100 \mathrm{ml}$. The carbol fuschin solution is prepared by mixing basic fuschin in the alcohol. To this mixture, water is added slowly while shaking. $8 \mathrm{ml}$ of phenol, which is melted in a water bath at $56^{\circ} \mathrm{C}$, is added to the stain with a pipette.

2. Decolorizer: It contains $5 \%$ aqueous sulfuric acid.

3. Counterstain: It contains methylene blue, $0.3 \mathrm{~g}$ and distilled water, $100 \mathrm{ml}$. It is prepared by mixing methylene blue with distilled water.

The procedure of hot modified acid-fast staining method consists of following steps:

- A fecal smear is made on glass slide. It is fixed by heat by passing it over the Bunsen flame repeatedly for 3-5 min.

- The heat-fixed slide is flooded with carbol fuschin. The slide is heated intermittently till carbol fuschin starts steaming. Slide is allowed to stain for 7-9 min. More carbol fuschin is added to prevent drying.

- The smear is washed with distilled water and decolorized with 5\% $\mathrm{H}_{2} \mathrm{SO}_{4}$ for $1 \mathrm{~min}$

- The smear is then washed with distilled water, and methylene blue is added for $1 \mathrm{~min}$ as the counterstain.

- Finally, the smear is washed with distilled water; water is drained off and dried and examined under the microscope using oil immersion objective.

The acid-fast oocysts of Cryptosporidium, Isospora, and Cyclospora stained red with their typical morphological features. Non-acid fast background stains blue with methylene blue.

\section{RESULTS}

Various enteric parasites detected in HIV-positive patients and HIV-negative individuals are shown in Table 1. For the purpose of statistical analysis, the enteric parasites are divided into the coccidian group (Cryptosporidium + I. belli) and conventional enteric parasites (Entamoeba histolytica, Giardia intestinalis, and Ascaris lumbricoides). There is a statistically significant relationship between the HIV status and enteric parasites. Enteric parasitic infection is more common in HIV-positive individuals (Fisher's exact test, $\mathrm{p}=0.044$, two-sided).

Various enteric parasites detected in HIV-positive patients with/ without diarrhea and in HIV-negative individuals are shown in Table 2.

Table 1: Enteric parasites detected from HIV-positive patients and control group

\begin{tabular}{lll}
\hline Parasite species & $\begin{array}{l}\text { HIV positive } \\
\mathbf{n = 7 5}(\mathbf{\%})\end{array}$ & $\begin{array}{l}\text { HIV negative } \\
\mathbf{n = 5 0}(\%)\end{array}$ \\
\hline Cryptosporidium & $06(8)$ & 00 \\
Isospora & $01(1.33)$ & 00 \\
E. histolytica & $02(2.67)$ & $03(6)$ \\
G. intestinalis & $03(4)$ & $02(4)$ \\
A. lumbricoides & $01(1.33)$ & $01(2)$ \\
Total (\%) & $13(17.3)$ & $06(12)$ \\
\hline
\end{tabular}

E. histolytica: Entamoeba histolytica, G. intestinalis: Giardia intestinalis, A. lumbricoides: Ascaris lumbricoides 
Table 2: Enteric parasites in HIV-positive patients with/without diarrhea and in HIV-negative individuals

\begin{tabular}{llll}
\hline Parasite species & HIV positive with diarrhea $(\mathbf{n = 1 8})$ & HIV positive without diarrhea $(\mathbf{n = 5 7})$ & HIV negative individuals (n=50) \\
\hline Cryptosporidium & 06 & 00 & 00 \\
Isospora & 01 & 00 & 00 \\
E. histolytica & 01 & 01 & 03 \\
G. intestinalis & 01 & 02 & 02 \\
A. lumbricoides & 00 & 01 & 01 \\
Total & 09 & 04 & 06 \\
\hline
\end{tabular}

E. histolytica: Entamoeba histolytica, G. intestinalis: Giardia intestinalis, A. lumbricoides: Ascaris lumbricoides

Enteric parasitic infection is more common in HIV-positive patients with diarrhea than HIV-positive patient without diarrhea or HIV-negative individuals. There is a statistically significant relationship between diarrhea and enteric parasites in HIV-infected patients (Fisher's exact test, $\mathrm{p}=0.021$, two-sided).

Details of the HIV-positive patients pertaining to occupation, sexuality, marital status, and other risk behaviors are recorded in Table 3. Promiscuity appears to be the highest risk factor in HIV-positive group.

Association of intestinal parasitic infection and ART is shown in Table 6. It is shown that coccidian parasitic infection is more common in patients without ART. However, no statistically significant relationship was found between ART status and enteric parasites (Fisher's exact test, $\mathrm{p}=0.286$, two-sided).

\section{DISCUSSION}

The present study documents that infection with enteric parasites is common in the HIV-positive patients having diarrhea in Kadapa, Andhra Pradesh. Of 75 HIV-seropositive cases studied, 18 (24\%) cases had diarrhea (Table 2). Detection of enteric parasites from HIV-positive patients having diarrhea was significantly higher $(9 / 18,50 \%)$ and statistically significant (Fisher's exact test, $\mathrm{p}=0.021$ ), compared to the patients without diarrhea (7.01\%) and HIV-negative patients (12\%). A similar study conducted by Talib and Singh where the isolation rate of enteric parasites from HIV-positive patients with diarrhea was $45.8 \%(11 / 24)$ [1]. Prasad et al. isolated 73\% (19/26) of the enteric pathogens from the HIV-positive patients with diarrhea [3]. In both of these studies, the presence of enteric parasites in HIV-positive patients without diarrhea is significantly low, as in our study. Kumar et al. isolated 36 enteric pathogens from 100 HIV-positive patients having diarrhea (36\%), whereas in the same study, isolation rate of enteric parasites from HIV-positive patients without diarrhea was $14 \%$ (7/50) [2]. However, a similar study by Mukhopadhya et al. documented that infection with enteric pathogens is common in southern Indian HIV-positive patients, regardless of the presence of diarrhea, and the overall isolation of enteric pathogens from stool was similar in patients with or without diarrhea [4].

Figures from various studies demonstrate striking geographic variations in the prevalence of individual pathogens in HIV-infected patients. These variations may relate both to the prevalence of pathogens within the community and to drugs used prophylactically in patients with HIV infections. Isolation rates, in this study (Table 1) of Cryptosporidium (8\%, reported rates 6-37\%), Giardia (4\%, reported rates $1-11 \%)$, and $I$. belli $(1.33 \%$, reported rates $0-3 \%)$ from HIVpositive patients, were within the ranges reported from elsewhere. In some recent studies, the isolation rate of $I$. belli has shown an increasing trend - Mukhopadhya et al. (18\%) [4], Kumar et al. (13.7\%) [2], and Prasad et al. (27\%) [3], compared with the previous ones - Talib and Singh (1.25\%) and Ballal et al. (2.86\%). The high rate of infection with I. belli poses a threat to HIV-positive patients. The actual rate of this infection in immuno-compromised individuals and AIDS patients is likely to be underestimated due to asymptomatic shedding of oocysts and treatment with trimethoprim-sulfamethoxazole for other infections in AIDS patients (Pneumocystis carinii pneumonia), which may confer some protection against this parasite.
Table 3: Risk factors, marital status, and occupation of 75 HIV-positive patients

\begin{tabular}{ll}
\hline Marital status & \\
Married & 52 \\
Unmarried & 23 \\
Risk factors & \\
Promiscuity & 21 \\
Blood transfusion & 08 \\
I.V drug users & 01 \\
Others (previous operation, spouse of HIV (+) person) & 28 \\
Occupation & \\
Driver & 06 \\
Laborer & 31 \\
Vendor & 15 \\
Student & 04 \\
Others & 19 \\
Circumcision in male & \\
Circumcised & 06 \\
Uncircumcised & 49 \\
\hline
\end{tabular}

Table 4: HIV positive Total 55 males and 20 female age group of 15 to 55 selected in this indiviaual parasites isolated in males 04 and females 02

\begin{tabular}{llllll}
\hline \multirow{2}{*}{ Age (years) } & \multicolumn{2}{l}{ Total number of patients } & & \multicolumn{2}{c}{ Parasites detected } \\
\cline { 2 - 3 } \cline { 5 - 5 } & Male & Female & & Male & Female \\
\hline $15-25$ & 19 & 04 & 02 & 01 \\
$26-35$ & 16 & 03 & 02 & 03 \\
$36-45$ & 11 & 08 & 02 & 02 \\
$46-55$ & 08 & 05 & 01 & 00 \\
$>55$ & 01 & 00 & 00 & 00 \\
Total & 55 & 20 & 07 & 06 \\
\hline
\end{tabular}

Table 5: HIV negative total 34 number of males and 16 females in age group of 18 to60, and individual isolated parasite males 04 and females 02

\begin{tabular}{llllll}
\hline \multirow{2}{*}{ Age (years) } & \multicolumn{2}{l}{ Total number of patients } & & \multicolumn{2}{l}{ Parasites detected } \\
\cline { 2 - 3 } \cline { 5 - 6 } & Male & Female & & Male & Female \\
\hline $18-30$ & 12 & 03 & & 02 & 00 \\
$31-40$ & 09 & 04 & 01 & 01 \\
$41-50$ & 07 & 06 & 00 & 01 \\
$51-60$ & 06 & 03 & 01 & 00 \\
Total & 34 & 16 & 04 & 02 \\
\hline
\end{tabular}

Table 6: Association of enteric parasites and ART

\begin{tabular}{lll}
\hline Parasites & On ART & Without ART \\
\hline Cryptosporidium & 02 & 04 \\
I. belli & 00 & 01 \\
E. histolytica & 01 & 01 \\
G. intestinalis & 02 & 01 \\
A. lumbricoides & 01 & 00 \\
Total & 06 & 08 \\
\hline
\end{tabular}

ART: Antiretroviral therapy, I. belli: Isospora belli, E. histolytica: Entamoeba histolytica, G. intestinalis: Giardia intestinalis, A. lumbricoides: Ascaris lumbricoides 
The detection rate of Cryptosporidium oocysts from HIV-positive patients in our study $(8 \%)$ is in accordance with the other studies (6-37\%). All the stool samples positive for Cryptosporidium oocysts were diarrheal. Cryptosporidium is also the most prevalent parasite $(6 / 13,46.1 \%)$ in our study group of HIV-positive patients. No Cryptosporidium oocysts were detected from the HIV-positive patients without diarrhea or from HIV-negative individuals. This finding suggests that intestinal infestation with Cryptosporidium spp is associated with diarrhea in HIVpositive patients. A similar study conducted by Mukhopadhya et al. and Kumar et al. where the isolation rate of Cryptosporidium oocysts from HIV-positive patients without diarrhea was $6 \%$ and $8 \%$, respectively. However, Talib and Singh also documented that all the Cryptosporidium positive stool samples were diarrheal.

In our study group, maximum patients were laborer (41\%). Promiscuity appeared to be the highest risk factor among the study group. Among the 55 male patients, only 6 patients had circumcision while 49 patients were uncircumcised. As the socioeconomic status of our study group was very poor, so we were unable to get information on the $\mathrm{CD}_{4}+$ count of the patients. For four patients, the $\mathrm{CD}_{4}+\mathrm{T}$ lymphocyte count done elsewhere was recorded and all of them were above $200 / \mu l$. Hence, the relation between $\mathrm{CD}_{4}+$ count and presence of intestinal parasites could not be established. The earlier study showed that intestinal opportunistic protozoal infection and diarrhea are more common in patients with low $\mathrm{CD}_{4}+$ count [11-13].

Another important finding in our study was that all the microscopy positive stool samples for Cryptosporidium oocysts appeared dark green to olive green in color and watery to semiformed in consistency. The same findings were observed by Nagamani et al. in their study on "molecular characterization of Cryptosporidium between 2003 and 2006" [14]. It was also observed in a few patients that as these samples became negative on microscopy, the character of stool changed from watery green to formed yellow color [15].

We were unable to identify any mixed enteric infection in our study though there are reports about mixed or coinfection in few other studies. Shah et al. reported a case of AIDS from Gujarat with coinfection with Cryptosporidium, Isospora, and Strongyloides stercoralis [16]. Prasad et al. identified two mixed infections, I. belli with S. stercoralis in one and the other is Cryptosporidium with E. histolytica [3,17]. Kumar et al. reported 3 cases of mixed infection with enteric pathogens in HIV-positive patients which includes 2 cases of mixed infection with Cryptosporidium and I. belli and one case of Cryptosporidium with Ancylostoma duodenale $[2,18]$. The mixed infections depend on multiple factors such as severe immunosuppression, poor sanitary condition, and geographical variations.

\section{SUMMARY AND CONCLUSION}

1. The study period of 1 year and 8 months revealed that it is difficult to locate/identify a HIV-positive person in a place like Kadapa on account of confidentiality/lack of awareness among the population.

2. Having identified the HIV-positive cases, a collection of stool samples from the patients also needed counseling and intervention by the respective clinicians, especially when patients were not having any symptoms.

3. Follow-up of the HIV-positive cases is difficult as they lose contact with the clinicians and not traceable after being discharged from the hospital.

4. As there was difficulty in identifying the HIV-positive cases, having located a case we collected stool samples from the patients.

5. Detection of intestinal parasites is $17.3 \%$ in HIV-positive patients and $12 \%$ in HIV-negative individuals.
6. In HIV-positive cases with diarrhea, detection of intestinal parasites is $50 \%$, whereas in HIV-positive cases without diarrhea, it is only $7.01 \%$.

7. Coccidian parasites Cryptosporidium and Isospora are detected only in HIV-positive cases with diarrhea. They are not detected in HIVpositive cases without diarrhea or HIV-negative individuals.

\section{AUTHOR CONTRIBUTION}

4500 paid, November 9th, Ref No 731221014341,

\section{CONFLICT OF INTEREST}

Nill

\section{SOURCE OF FUND}

Self

\section{REFERENCES}

1. Talib SH, Singh J. A study of opportunistic enteric parasites in $80 \mathrm{HIV}$ seropositive parients. Indian J Pathol Microbiol 1998;41:31-7.

2. Kumar SS, Ananthan S, Saravanan P. Role of coccidian parasites in causation of diarrhoea in HIV infected patients in Chennai. Indian $\mathrm{J}$ Med Res 2002;116:85-9.

3. Prasad KN, Nag VL, Dhole TN, Ayyagari A. Identification of enteric pathogens in HIV-positive patients with diarrhoea in Northern India. J Health Popul Nutr 2000;18:23-6.

4. Mukhopadhya A, Ramakrishna BS, Kang G, Anna B. Enteric pathogens in southern Indian HIV-infected patients with and without diarrhea. Indian J Med Res 1999;109:85-9.

5. Hailemariam G, Kassu A, Abebe G, Abate E, Damte D, Mekonnen E, et al. Intestinal parasitic infections in HIV/AIDS and HIV seronegative individuals in a teaching hospital, Ethiopia. Jpn J Infect Dis 2004;57:41-3.

6. Ballal M, Prabhu T, Chandran A, Shivananda PG. Cryptosporidium and Isospora belli diarrhoea in immunocompromised hosts. Indian J Cancer 1999;36:38-42.

7. Kumar SS, Lakshmi P, Ananthan S. Intestinal parasitic infection in HIV infected patients in Chennai. Indian J Med Microbiol 2002;20:88-91.

8. Chatterjee KD. Parasitology in Relation to Clinical Medicine. $12^{\text {th }}$ ed. New Delhi: Chartterjee Medical Publishers; 1981

9. Parija SC. Text book of Medical Parasitology. $3^{\text {rd }}$ ed. Chennai: All India Publishers \& Distributors; 2003.

10. Garcia LS, Bruckner DA, Brewer TC, Shimizu RY. Techniques for the recovery and identification of Cryptosporidium oocysts from stool specimens. J Clin Microbiol 1983;18:185-90.

11. Hyun G, Lower FC. AIDS and the urologist. Urol Clin North Am 2003;30:101-9.

12. Muthusamy D, Rao SS, Ramani S, Monica B, Banerjee I, Abraham OC, et al. Multilocus genotyping of Cryptosporidium sp. Isolates from human immunodeficiency virus-infected individuals in south India. J Clin Microbiol 2006;44:632-4.

13. Attili SV, Gulati AK, Singh VP, Varma DV, Rai M, Sundar S, et al. Diarrhea, CD4 counts and enteric infections in a hospital - Based cohort of HIV-infected patients around Varanasi, India. BMC Infect Dis 2006;6:39.

14. Nagamani K, Pavuluri PR, Gyaneshwari M, Prasanthi K, Rao MI, Saxena NK, et al. Molecular characterisation of Cryptosporidium: An emerging parasite. Indian J Med Microbiol 2007;25:133-6.

15. Nagamani K, Rajkumari A, Gyaneshwari. Cryptosporidiosis in a tertiary care hospital in Andhra Pradesh. Indian J Med Microbiol 2001;19:215-6.

16. Shah UV, Purohit BC, Chandralekha D, Mapara MH. Coinfection with Cryptosporidium, Isospora and S. stercoralis in a patient with AIDS - A case report. Indian J Med Microbiol 2003;21:137-8.

17. Tambekar. Antimicrobial potential treatment in enteric infection. Int $\mathrm{J}$ Pharm Pharm Sci 2010;2:176-9.

18. Divyashanthi CM. Prevalence and anti-microbial susceptibility pattern in admitted patients. Int J Pharm Pharm Sci 2015;7:186-90. 